\title{
Efficacy and Safety of $1 \%$ Terbinafine Film-Forming Solution in Chinese Patients with Tinea Pedis: A Randomized, Double- Blind, Placebo-Controlled, Multicenter, Parallel-Group Study
}

\author{
Ruo Yu Li • A. P. Wang $\cdot$ J. H. Xu • L. Y. Xi • \\ M. H. Fu • M. Zhu • M. L. Xu • X. Q. Li • \\ W. Lai • W. D. Liu • X. Y. Lu • Z. Q. Gong
}

Published online: 30 January 2014

(C) The Author(s) 2014. This article is published with open access at Springerlink.com

\begin{abstract}
Background and objectives Superficial fungal skin infections are treated using topical antifungals. The aim of this study was to demonstrate the efficacy of a single application of $1 \%$ terbinafine film-forming solution (FFS) versus placebo for the treatment of tinea pedis in the Chinese population.

Methods Six centers in China randomized 290 patients in a $1: 1$ ratio to receive either $1 \%$ terbinafine FFS or FFS vehicle (placebo) once on the affected foot/feet. Efficacy assessments included microscopy and mycologic culture, and assessing clinical signs and symptoms at baseline, and
\end{abstract}

ClinicalTrial.gov registration number: NCT01433107.

R. Y. Li $(\bowtie) \cdot$ A. P. Wang

Dermatology Department, Peking University First Hospital, 8\# Xishiku Street, Xicheng District, Beijing 100034, China e-mail: 1ry0660@gmail.com

J. H. Xu · M. Zhu

Huashan Hospital, Fudan University, Shanghai, China

L. Y. Xi · X. Q. Li

Dermatology Department, Sun Yat-sen Memorial Hospital, Sun Yat-sen, Guangzhou, China

M. H. Fu - W. D. Liu

Institute of Dermatology, Chinese Academy of Medical

Sciences, Nanjing, Jiangsu, China

M. L. Xu $\cdot$ X. Y. Lu

Dermatology Department, Peking University Third Hospital, Beijing, China

W. Lai · Z. Q. Gong

Dermatology Department, The Third Affiliated Hospital of Sun

Yat-sen University, Guangzhou, China at weeks 1 and 6 after the topical treatment. All adverse events were recorded.

Results At week 6,1\% terbinafine FFS was superior to placebo for effective treatment rate (63 vs. $8 \%$ ); clinical cure ( 30 vs. $6 \%$ ); mycological cure ( 86 vs. $12 \%$ ); negative microscopy (90 vs. $24 \%$ ); and negative mycological culture (90 vs. $27 \%$ ): all $p \leq 0.001$ and clinically relevant. At week $6,1 \%$ terbinafine FFS was clinically superior to placebo for the absence of: erythema (69 vs. $29 \%$ ); desquamation (33 vs. $8 \%$ ); and pruritus (70 vs. $30 \%$ ): all $p \leq 0.001$ and clinically relevant. At week 6 , differences in the average total signs and symptoms scores were significantly lower for $1 \%$ terbinafine FFS versus placebo $(p \leq 0.001)$. Both $1 \%$ terbinafine FFS and placebo were safe and well tolerated based on adverse events and investigator and patient assessments.

Conclusions This double-blind, randomized, multicenter study demonstrated one single topical application of $1 \%$ terbinafine FFS was safe and effective in the treatment of tinea pedis in the Chinese population.

\section{Introduction}

Tinea pedis (athlete's foot) is becoming increasingly prevalent worldwide because of various factors including changes in lifestyle and incidence of various diseases. Its prevalence is now more than $50 \%$ of the population in Northern and Central Europe, approximately $25 \%$ in the Mediterranean, and $28 \%$ in Singapore; an incidence of $39 \%$ has also been reported in North Western China [1-3].

Tinea pedis is generally treated with the topical application of various antifungal creams, gels, sprays, liquids, and powders. Topical terbinafine has been highly successful in treating tinea pedis. A single application of $1 \%$ 
terbinafine film forming solution (FFS) has been shown to be as safe, and superior in efficacy, to placebo in a multicenter European study in tinea pedis. The relapse/reinfection rate after 3 months was similar to that using $1 \%$ terbinafine cream for 7 days [4].

One percent terbinafine FFS is an alcohol solvent formulation that evaporates upon application to the skin, leaving a smooth, almost invisible film that delivers terbinafine to the skin. After a single application, high levels of $1 \%$ terbinafine penetrate the stratum corneum where dermatophytes are located and fungicidal levels of terbinafine remain there for up to 13 days [5].

Here, we report the results of a randomized, doubleblind, placebo-controlled, multicenter, parallel-group study of the efficacy and safety of $1 \%$ terbinafine FFS in Chinese patients with tinea pedis.

\section{Patients and Methods}

\subsection{Patients}

This was a randomized, double-blind, placebo-controlled, multicenter, parallel-group study conducted at six investigational centers in China. Ethical committee approval was obtained at each study center. Patients who were consulted for tinea pedis infection in the study centers were questioned regarding their tinea pedis history and their eligibility was confirmed against the following inclusion criteria: male or female individual aged $\geq 18$ years with clinical tinea pedis infection (lesions between the toes with possible extension to lateral borders of foot and sole), total signs and symptoms score $\geq 3$, and positive microscopy [potassium hydroxide $(\mathrm{KOH})$ wet mount] from the target foot prior to one single topical treatment. Patients with positive microscopy at baseline were to be considered as a delayed exclusion if the result of the culture of the mycologic sample taken at the baseline visit was negative (or positive, but not for a dermatophyte). Only patients who were able to provide written, informed consent were included in the study. The study was registered on the Clinicaltrials.gov website (registration number: NCT01433107).

The planned number of patients was 286 , randomized in a 1:1 ratio to receive one single dose of either $1 \%$ terbinafine FFS or FFS vehicle (placebo) applied to the affected foot (or feet) on day 1.

\subsection{Randomization}

A randomization list was produced by using a validated system that automates the random assignment of treatment to randomization numbers. At the randomization/treatment visit (day 1), each subject who fulfilled all the inclusion/ exclusion criteria was given the lowest available number on the randomization list available at the study site. This number assigned them to one of the two treatment arms.

\subsection{Treatment Blinding}

Subjects, investigator staff, persons performing the assessments, study monitors, and data analysts were blinded to the identity of the treatment from the time of randomization until database lock. This was achieved by keeping randomization data strictly confidential until the time of unblinding, and by the use of study treatments that were all identical in packaging, labeling, schedule of administration, appearance, and odor.

\subsection{Study Visits}

Follow-up visits to the study center took place 1 and 6 weeks after treatment. Even if both feet were infected, only one foot was to be assessed for efficacy (the target foot). The study was completed when all patients completed all visits up to the week- 6 final visit and when all mycologic evaluations up to the week-6 visit were available. Adverse events (AEs) were collected throughout the study.

\subsection{Assessments}

Mycologic samples were taken at baseline and at weeks 1 and 6 and assessed by microscopy (KOH wet mount) and by culture. Mycologic samples were taken from inter-digital lesions with the most severe signs and symptoms of disease and pooled for $\mathrm{KOH}$ wet mount examination and culture. Mycologic cure was defined by negative $\mathrm{KOH}$ microscopy and negative mycologic culture. Clinical signs and symptoms scores were assessed at the baseline visit and at weeks 1 and 6. Clinical signs and symptoms including desquamation (scaling), erythema, pruritus, incrustation (crusting), pustules, and vesiculation were evaluated by the investigator and recorded at every visit using a 4 -point scale $(0=$ absent, $1=$ mild, $2=$ moderate, 3 = severe). Clinical cure was defined as all assessments of signs and symptoms scored as absent. Complete cure was defined as both mycologic cure and clinical cure.

\subsection{Study Objectives}

The primary efficacy variable was the rate of effective treatment at week 6, using the following definition: mycologic cure and total sum of signs and symptoms score $\leq 2$, provided that individual severity scores for incrustation, pustules, and vesiculation were absent (0), and individual severity scores for desquamation, erythema, and 
pruritus were absent or mild $(\leq 1)$. The secondary objectives were to evaluate the safety profile of $1 \%$ terbinafine FFS; mycologic cure rate at weeks 1 and 6 and effective treatment rate at week 1 .

At weeks 1 and 6, the investigator assessed efficacy and safety of the treatments. The patients assessed local tolerability at week 6 , on a 5-point scale (very good, good, moderate, poor, and very poor). If both feet were treated, both feet were to be assessed equally for safety.

\subsection{Statistical Analysis}

The intent-to-treat analysis set (ITT) was defined as all randomized patients regardless of whether or not they had been treated and regardless if they were delayed exclusions or not. A full analysis set (FAS) was also defined, which included all randomized patients who had been treated and who were not delayed exclusions. In both ITT and FAS, patients with missing mycology results or missing clinical signs and symptoms scores were treated as "failure" in the analysis of the main efficacy variable. The per-protocol set (PPS) was defined as patients included in the FAS, who provided efficacy results at week 6 and who had no major protocol violation that could impact the efficacy evaluation. Efficacy analysis was conducted on the ITT, FAS, and PPS populations, and analysis of the FAS was considered the primary efficacy analysis.

The two treatment groups were compared with respect to the effective treatment rate using the Cochran-MantelHaenszel $(\mathrm{CMH})$ test of general association stratified by centers. A supportive analysis was completed using a logistic regression model fitted to compare the effective treatment rate between the two treatment groups. The influence of the following covariates was explored prior to including the treatment effect in the model: total signs and symptoms score at baseline, type of dermatophytes at baseline, and extension to sole or lateral side and center. The odds ratio and $95 \%$ confidence interval for the treatment effect was tabulated. Secondary endpoints defined as rates (clinical cure, mycologic cure, and complete cure rates) were analyzed similarly to the primary efficacy endpoint analysis.

Individual clinical signs and symptoms scores were summarized by treatment group. Differences between treatment groups were analyzed using a CMH test with rank scores, stratified by center and baseline level. The total clinical signs and symptoms score was treated as a continuous variable and summarized appropriately. Differences between treatment groups were analyzed using an analysis of covariance with center and baseline score as a covariate. Adverse events and other secondary endpoints were summarized descriptively.

\section{Results}

\subsection{Baseline Characteristics}

The baseline demographic and disease characteristics of the two treatment groups were similar (Table 1). A total of 290 patients were enrolled and randomized, 145 each to $1 \%$ terbinafine FFS and placebo, of which 246 patients ( $85 \%$ ) completed the study, and 44 patients ( $15 \%$ in total, $19 \%$ in the $1 \%$ terbinafine FFS group, and $12 \%$ in the placebo group) discontinued from the study prior to week 6 . The primary reason for discontinuation was because of mycologic test procedure results $(14 \%)$, i.e., delayed exclusions that were not followed up until week 6 . In addition, two patients discontinued because of protocol deviations, one patient discontinued because of withdrawal of consent, and one patient was lost to follow-up. Because of the delayed exclusions, 237 patients were included in the FAS, 115 in the $1 \%$ terbinafine FFS group, and 122 in the placebo group.

The epidemiologic pattern of disease observed was representative of a typical tinea pedis population: over $85 \%$ of patients had bilateral lesions and $50 \%$ had lesion extensions on the sole(s) and/or lateral area(s) of the feet. Trichophyton rubrum was identified in $68 \%$ of patients randomized in each of the treatment groups, followed by Trichophyton mentagrophytes, which was prevalent in $11 \%$ of the $1 \%$ terbinafine FFS randomized patients and in $17 \%$ of the placebo randomized patients. The median duration of current tinea pedis was around 12 weeks. Prior to the study the patients had an average of three episodes per year. These previous episodes were moderate to severe for more than $75 \%$ of the patients.

\subsection{Treatment Follow-Up at Week 6}

Figure 1 illustrates the results of treatment at week 6 . At Week 6 , over $63 \%, 86 \%$, and $30 \%$, of patients in the $1 \%$ terbinafine FFS group experienced effective treatment, mycologic cure, and clinical cure, respectively, compared with $8 \%, 12 \%$, and $6 \%$, in the placebo group, respectively. All three differences were statistically significant $(p<0.001)$ and clinically relevant. The superiority of $1 \%$ terbinafine FFS over placebo shown in the primary efficacy analysis conducted on the FAS population was confirmed both in the ITT and PPS populations.

\subsection{Mycologic Evaluations at Weeks 1 and 6}

At week 1, a total of $49 \%$ and $81 \%$ of patients in the $1 \%$ terbinafine FFS group experienced negative microscopy and negative mycologic culture, respectively, compared with 26 $\%$ and $24 \%$ in the placebo group, respectively. Both 
Table 1 Patient and disease characteristics

\begin{tabular}{|c|c|c|c|}
\hline Characteristic/statistic/category & $1 \%$ terbinafine FFS $(n=145)$ & Placebo $(n=145)$ & Total $(n=290)$ \\
\hline \multicolumn{4}{|l|}{ Age (years) } \\
\hline Mean \pm SD & $35.27 \pm 12.805$ & $34.05 \pm 11.936$ & $34.66 \pm 12.372$ \\
\hline Range & $18.5-82.3$ & $18.6-84.1$ & $18.5-84.1$ \\
\hline \multicolumn{4}{|l|}{ Sex, $n(\%)$} \\
\hline Male & $95(65.5)$ & $99(68.3)$ & $194(66.9)$ \\
\hline Female & $50(34.5)$ & $46(31.7)$ & $96(33.1)$ \\
\hline \multicolumn{4}{|l|}{ Sites of tinea pedis lesion, $n(\%)$} \\
\hline Unilateral & $23(15.9)$ & $17(11.7)$ & $40(13.8)$ \\
\hline Bilateral & $122(84.1)$ & $128(88.3)$ & $250(86.2)$ \\
\hline Inter-digital space (s) only, $n(\%)$ & $72(49.7)$ & $71(49.0)$ & $143(49.3)$ \\
\hline Extension to sole/lateral surfaces, $n(\%)$ & $73(50.3)$ & $74(51.0)$ & $147(50.7)$ \\
\hline Positive for dermatophyte & $115(79.3)$ & $122(84.1)$ & $237(81.7)$ \\
\hline \multicolumn{4}{|c|}{ Species identified on culture (all collection areas), $n(\%)$} \\
\hline Trichophyton rubrum & $98(67.6)$ & $98(67.6)$ & $196(67.6)$ \\
\hline Trichophyton mentagrophytes & $16(11.0)$ & $25(17.2)$ & $41(14.1)$ \\
\hline Trichophyton tonsurans & $0(0)$ & $0(0)$ & $0(0)$ \\
\hline Epidermophyton floccosum & $1(0.7)$ & $0(0)$ & $1(0.3)$ \\
\hline \multicolumn{4}{|c|}{ Duration of current tinea pedis episode (weeks), $n(\%)$} \\
\hline Number of patients & 137 & 142 & 279 \\
\hline Mean $\pm \mathrm{SD}$ & $17.65 \pm 21.487$ & $20.67 \pm 43.707$ & $19.19 \pm 34.598$ \\
\hline Range & $0.4-176.0$ & $0.1-487.3$ & $0.1-487.3$ \\
\hline$<2$ & $13(9.0)$ & $13(9.0)$ & $26(9.0)$ \\
\hline $2-<4$ & $14(9.7)$ & $20(13.8)$ & $34(11.7)$ \\
\hline $4-<8$ & $25(17.2)$ & $18(12.4)$ & $43(14.8)$ \\
\hline $8-<26$ & $54(37.2)$ & $59(40.7)$ & $113(39.0)$ \\
\hline $26-<52$ & $26(17.9)$ & $24(16.6)$ & $50(17.2)$ \\
\hline$\geq 52$ & $7(4.8)$ & $9(6.2)$ & $16(5.5)$ \\
\hline Missing & $6(4.1)$ & $2(1.4)$ & $8(2.8)$ \\
\hline \multicolumn{4}{|c|}{ Average number of tinea pedis episodes per year } \\
\hline Number of patients & 145 & 145 & 290 \\
\hline Mean \pm SD & $3.3 \pm 3.15$ & $3.0 \pm 2.55$ & $3.2 \pm 2.87$ \\
\hline Range & $0-20$ & $0-15$ & $0-20$ \\
\hline \multicolumn{4}{|l|}{ Severity of tinea pedis, $n(\%)$} \\
\hline Mild & $19(13.1)$ & $19(13.1)$ & $38(13.1)$ \\
\hline Moderate & $62(42.8)$ & $78(53.8)$ & $140(48.3)$ \\
\hline Severe & $48(33.1)$ & $35(24.1)$ & $83(28.6)$ \\
\hline Missing & $16(11.0)$ & $13(9.0)$ & $29(10.0)$ \\
\hline \multicolumn{4}{|c|}{ Tinea pedis episodes responsive to systemic or topical antifungal drugs, $n(\%)$} \\
\hline No & $0(0)$ & $0(0)$ & $0(0)$ \\
\hline Yes & $105(72.4)$ & $110(75.9)$ & $215(74.1)$ \\
\hline Missing & $40(27.6)$ & $35(24.1)$ & $75(25.9)$ \\
\hline
\end{tabular}

SD standard deviation

differences were statistically significant $(p<0.001)$ and clinically relevant. However, over time, $1 \%$ terbinafine FFS was even more effective than placebo. At week 6 , a total of $90 \%$ and $90 \%$ of patients in the $1 \%$ terbinafine FFS group experienced negative microscopy and negative mycological culture, respectively, compared with $24 \%$ and $27 \%$ in the placebo group, respectively. Both differences were statistically significant $(p<0.001)$ and clinically relevant.

Although the same proportion in the $1 \%$ terbinafine FFS group experienced negative microscopy and negative 


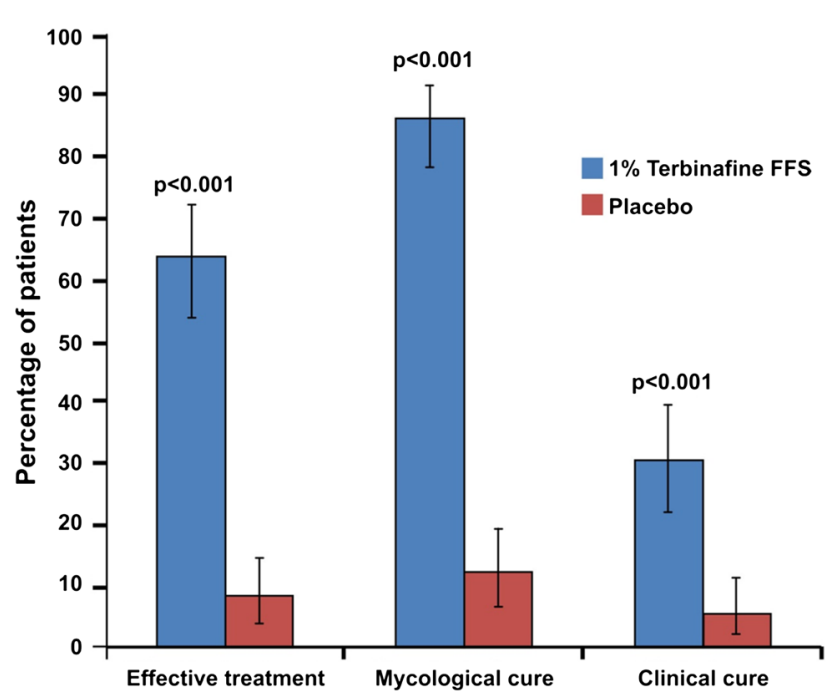

Fig. 1 Summary of treatment results at week 6, of $1 \%$ terbinafine film-forming solution (FFS) versus placebo, assessed by effective treatment, mycologic cure, and clinical cure (full analysis set)

mycologic culture, there was not a complete match of these results in the same subjects. That is to say that some subjects experienced negative microscopy but positive mycologic culture and vice versa. In general, mycologic culture results did match the direct microscopy results and there were fewer discrepancies in the terbinafine group (93\% matching) than in the placebo group $(73.8 \%)$. The small discrepancy in the terbinafine group reduced the mycologic cure rate to $86 \%$.

\subsection{Summary of Clinical Signs and Symptoms at Weeks 1 and 6}

Erythema, desquamation, and pruritus were the most frequent signs and symptoms at baseline. They were all present in more than $90 \%$ of the patients in each treatment group at baseline.

Figure 2 illustrates the signs and symptoms scores of erythema characteristics at baseline, and weeks 1 and 6 . At baseline and week 1 , the percentage of patients with mild, moderate, and severe ratings of erythema was similar between both treatment groups. However, over time, $1 \%$ terbinafine FFS was more effective than placebo. At week $6,69 \%$ of patients reported absence of erythema, compared with only $29 \%$ in the placebo group. This difference was statistically significant $(p<0.001)$. Interestingly, at week 6 , only $1 \%$ of patients reported severe erythema in the $1 \%$ terbinafine FFS group, compared with $7 \%$ in the placebo group.

Figure 3 illustrates the signs and symptoms scores of desquamation characteristics at baseline, and at weeks 1 and 6. At baseline, all patients experienced desquamation and the percentage of patients with mild, moderate, and

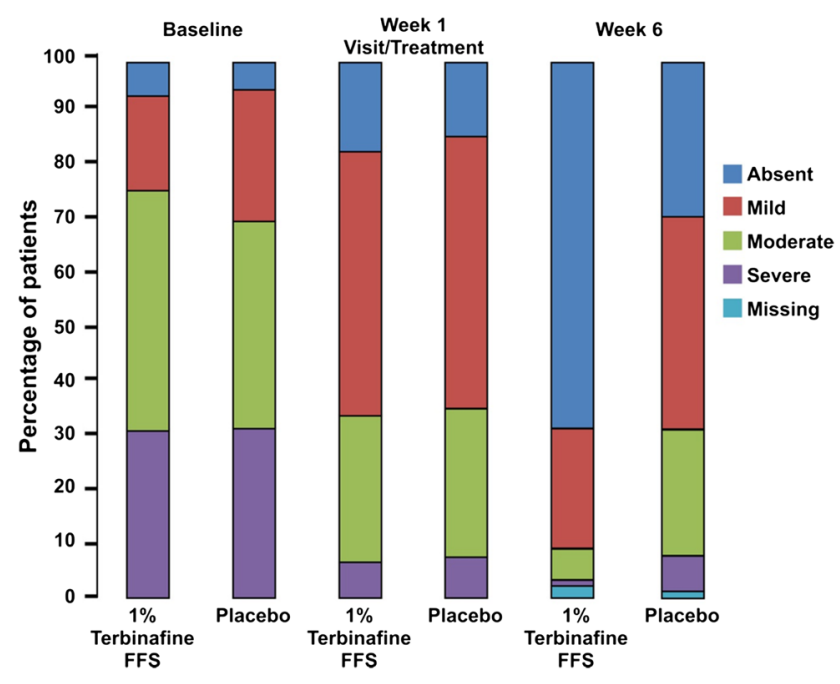

Fig. 2 Signs and symptoms scores of erythema at baseline and at weeks 1 and 6 , of $1 \%$ terbinafine film-forming solution (FFS) versus placebo (full analysis set). $p<0.001$ week 6 versus baseline

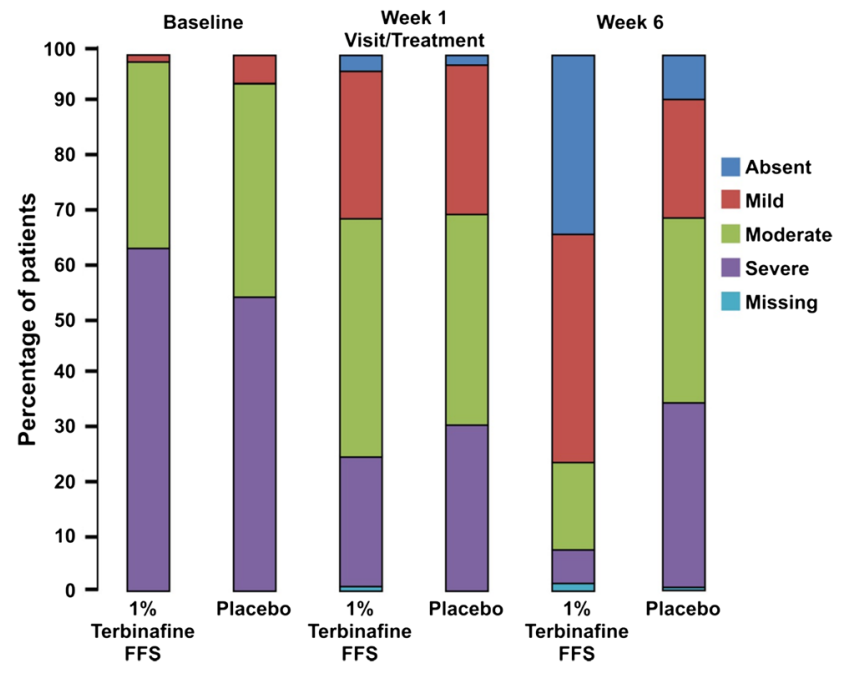

Fig. 3 Signs and symptoms scores of desquamation at baseline and at weeks 1 and 6 , of $1 \%$ terbinafine film-forming solution (FFS) versus placebo (full analysis set). $p<0.001$ week 6 versus baseline

severe ratings of desquamation was similar between both treatment groups, with the majority of patients experiencing severe or moderate desquamation (in $99 \%$ and $95 \%$ of patients in the $1 \%$ terbinafine FFS and placebo groups, respectively). At week 1, the majority of patients still experienced desquamation and the percentage of patients with mild, moderate, and severe ratings of desquamation was again similar between both treatment groups. However, over time, $1 \%$ terbinafine FFS was more effective than placebo. At week 6, $33 \%$ of patients reported absence of desquamation in the $1 \%$ terbinafine FFS group, compared with only $8 \%$ in the placebo group and the desquamation observed was mild in the $1 \%$ terbinafine FFS 
group, whereas it was still moderate to severe in the placebo group. Interestingly, at week 6 , only $6 \%$ of patients reported severe desquamation in the $1 \%$ terbinafine FFS group, compared with $34 \%$ in the placebo group. The difference in desquamation severity was statistically significant $(p<0.001)$.

Figure 4 illustrates the signs and symptoms scores of pruritus characteristics at baseline, and at weeks 1 and 6 . At baseline, the percentage of patients with mild, moderate, and severe ratings of pruritus was similar between both treatment groups, with the majority of patients experiencing severe or moderate pruritus (in $85 \%$ and $84 \%$ of patients in the $1 \%$ terbinafine FFS and placebo groups, respectively). However, over time, $1 \%$ terbinafine FFS was significantly more effective than placebo. At week 1 , about $60 \%$ of the patients still experienced pruritus; however, the percentage of patients reporting moderate to severe pruritus was almost twice that in the placebo group compared with the $1 \%$ terbinafine FFS group (19 vs. $34 \%$ ). At Week $6,70 \%$ of patients reported absence of pruritus in the $1 \%$ terbinafine FFS group, compared with only $30 \%$ in the placebo group. Mild and moderate pruritus was observed in $25 \%$ and $3 \%$ of patients in the $1 \%$ terbinafine group FFS, respectively, compared with $38 \%$ and $21 \%$ in the placebo group, respectively. Interestingly, at week 6 , no patients reported severe pruritus in the $1 \%$ terbinafine FFS group, compared with 13 patients $(11 \%)$ in the placebo group. The difference in pruritus severity was statistically significant $(p<0.001)$.

At week 1, the average total signs and symptoms scores were similar; 4.2 in 114 patients in the $1 \%$ terbinafine FFS group and 4.8 in 122 patients in the placebo group; but the difference nevertheless was statistically significant

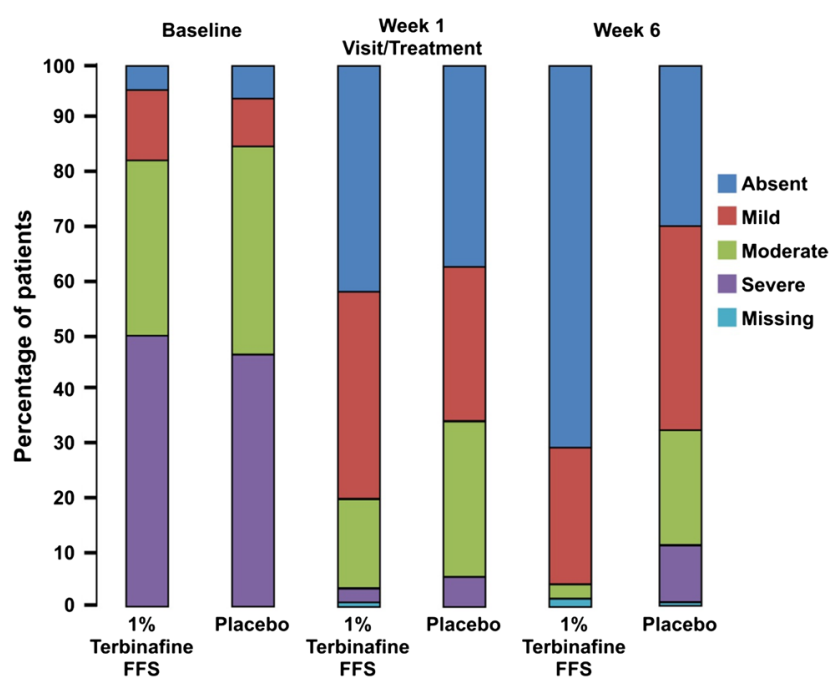

Fig. 4 Signs and symptoms scores of pruritus at baseline and at weeks 1 and 6 , of $1 \%$ terbinafine film-forming solution (FFS) versus placebo (full analysis set). $p<0.001$ week 6 versus baseline $(p=0.005)$. At week 6 , the average signs and symptoms score for $1 \%$ terbinafine FFS was much lower compared with placebo; 1.6 in 113 patients versus 4.7 in 121 patients, respectively. This difference was again statistically significant $(p<0.001)$.

The investigator global assessment of efficacy at week 6 was rated very good or good in $81 \%$ of patients in the $1 \%$ terbinafine FFS group, compared with $25 \%$ in the placebo group.

\subsection{Safety}

A total of 20 patients experienced at least one $\mathrm{AE}$ in the study; 9 in the $1 \%$ terbinafine FFS group and 11 in the placebo group. A total of 30 AEs were reported; 14 AEs in the $1 \%$ terbinafine FFS group and 16 AEs in the placebo group. All AEs were mild or moderate in severity. A total of 26 AEs were suspected to be related to study treatment; 13 AEs each in the $1 \%$ terbinafine FFS and placebo group. The most frequently reported AEs were application-site pain in six patients (4\%) each in the two treatment groups, and application-site pruritus in three patients $(2 \%)$ in the placebo group.

In addition, investigators' assessments of safety were similar between the two treatment groups; investigators rated the safety as very good or good, in $97 \%$ of patients in the $1 \%$ terbinafine FFS group and $99 \%$ in the placebo group. Furthermore, patient assessment of local tolerability was also rated similar between the two treatment groups for very good or good, in $95 \%$ of patients in the $1 \%$ terbinafine FFS group and $92 \%$ in the placebo group.

\section{Discussion}

This multicenter study demonstrated a single application of $1 \%$ terbinafine FFS to be statistically and clinically more efficacious than placebo in the treatment of tinea pedis in a Chinese population. The $1 \%$ terbinafine FFS was safe and well tolerated by over $90 \%$ of the patients.

The results of this study mirror those of a similar study carried out in European patients [4], although in the current study patients had a disease severity (total signs and symptoms at baseline/plantar involvement) much greater than in the European phase III study. The baseline average signs and symptoms score was three times higher in the Chinese population and plantar involvement much more common (in many cases extending onto the lateral aspect of the soles). In addition, the placebo response in this study was much lower than in the European study ( 8 vs. $17 \%$ ). Extension of tinea pedis to the lateral surfaces of the foot is often an indication of chronic infection and associated with greater disease severity. The severe disease in the Chinese 
population could possibly also explain the low placebo response for effective treatment at week 6. Statistically significant differences were recorded in patients treated with $1 \%$ terbinafine FFS compared with placebo for effective treatment, mycological cure, clinical cure, negative microscopy, and negative mycological culture.

As seen previously, T. rubrum predominated, followed by $T$. mentagrophytes $[4,6]$. Clinical signs and symptoms scores were lower for patients in the $1 \%$ terbinafine FFS group compared with placebo at both weeks 1 and 6 . The proportion of patients exhibiting mild, moderate, and severe erythema, desquamation, and pruritus decreased during the study in those treated with $1 \%$ terbinafine FFS, through week 1 to week 6, compared with placebo. Differences in the average total signs and symptoms scores were significantly lower for $1 \%$ terbinafine FFS than placebo at weeks 1 and 6 . Moreover, the higher ratings of $1 \%$ terbinafine FFS in the investigator assessments of efficacy at week 6 in comparison with placebo further highlighted the superiority of $1 \%$ terbinafine FFS over placebo.

The rate of delayed exclusions because of positive microscopy with negative culture $(14 \%)$ was similar to that in the European study (16\%) [4], demonstrating the experience of the investigators in diagnosing tinea pedis and the effectiveness of the mycology laboratories.

As reported previously [4], $1 \%$ terbinafine FFS is safe and well tolerated. The occurrence of AEs in this study was low, and all were mild and moderate in nature and did not give rise to any new safety concerns about $1 \%$ terbinafine FFS. Investigator assessment of safety and patient assessment of local tolerability were rated very good or good in more than $90 \%$ of patients in the $1 \%$ terbinafine FFS group and the placebo group, indicating no safety or tolerability concerns.

The proportion of patients with missing data for each of the reported parameters was very low. The ease of a singledose application encourages those with athlete's foot to be treated and virtually eliminates noncompliance, which is known to be widespread [7]. Half of the respondents in a consumer survey [8] reported using antifungal treatments for less than 13 days, despite most antifungals requiring 3-4 weeks of therapy. In addition, the survey respondents had experienced tinea pedis for an average of 7 years and $64 \%$ experienced more than four episodes a year. This may partly be because of the prevalence of fungal spores but possibly owing to incomplete eradication of the fungus by their lack of compliance with conventional multi-dose treatments. The current study shows that even with a fungicidal treatment that quickly achieves high levels of active ingredient in the stratum corneum, the effect increases with time, indicating that contact between the agent and the fungus needs to be maintained.
As well as waning interest in prolonged topical antifungal treatments, patients may also erroneously interpret relief of signs and symptoms as indicating eradication of the fungus. It is well known that fungal elements may be present in the skin without causing signs or symptoms $[9$, 10]. Application of antifungal treatment to the whole of the foot will treat both symptomatic and non-symptomatic areas. Hay et al. concluded that this is more likely to be undertaken by patients using terbinafine $1 \%$ FFS compared with multi-dose treatments [11].

Furthermore in relation to compliance, a meta-analysis found terbinafine $1 \%$ FFS to be non-inferior to other antifungals when used for many weeks of treatment. The data for terbinafine $1 \%$ FFS were compared with trials of azoles and even other allylamines (butenafine and naftifine) versus vehicle, used for several weeks. Single treatment with terbinafine was found to be superior to some azoles (clotrimazole and oxiconazole) [12].

\section{Conclusion}

This study confirms that $1 \%$ terbinafine FFS, applied as a single dose, is a highly effective, safe, and convenient treatment for tinea pedis in a Chinese population. The incentive to comply with this one-time treatment method is likely to have benefits for both patients and public health in general.

Acknowledgments Author contributions All authors participated in the conception, design, and implementation of the trials. All authors were involved in the interpretation of analyzed data and the decision to submit for publication.

Conflict of interest There were no conflicts of interest.

Funding statement This study was supported by Beijing Novartis Pharma Ltd. The manuscript was prepared by Dr. Pascal Mallefet, Global Medical Affairs, Novartis Consumer Health with editorial help from professional medical writers (Bilal Bham and Mike Matthews of TMC Pharma Services) paid for by Novartis.

Open Access This article is distributed under the terms of the Creative Commons Attribution Noncommercial License which permits any noncommercial use, distribution, and reproduction in any medium, provided the original author(s) and the source are credited.

\section{References}

1. Ameen M. Epidemiology of superficial fungal infections. Clin Dermatol. 2010;28:197-201.

2. Tan HH. Superficial fungal infections seen at the National Skin Centre, Singapore. Nihon Ishinkin Gakkai Zasshi. 2005;46:77-80.

3. Tao-Xiang N, Zhi-Cheng L, Sao-Mao W, Wen-Zhu L. Analysis of dermatomycoses in Lanzhou district of northwestern China. Mycopathologia. 2005;160:281-4. 
4. Ortonne JP, Korting HC, Viguié-Vallanet C, Larnier C, Savaluny E. Efficacy and safety of a new single-dose terbinafine $1 \%$ formulation in patients with tinea pedis (athlete's foot): a randomized, double-blind, placebo-controlled study. J Eur Acad Dermatol Venereol. 2006;20:1307-13.

5. de Chauvin MF, Viguié-Vallanet C, Kienzler JL, Larnier C. Novel, single-dose, topical treatment of tinea pedis using terbinafine: results of a dose-finding clinical trial. Mycoses. 2008;51:1-6.

6. Kemna ME, Elewski B. A US epidemiologic survey of superficial fungal diseases. J Am Acad Dermatol. 1996;35:539-42.

7. Meinhof W, Girardi M, Stracke A. Patient non-compliance in dermatomycosis. Dermatologica. 1984;169:57-66.

8. Rodriguez G. Athlete's foot: what a patient thinks. Usage and attitudes. Consumer research of 900 respondents in the UK, France and Germany. Novartis Consumer Research Presented at Expert Panel Conference, 2005, London.
9. Becerril-Chihu G, Bazam-Mora E, Lopez-Martinez R, Sosa-deMartínez C, RuizMaldonado R. How often are dermatophytes present in apparently normal versus scaly feet of children? Pediatr Dermatol. 1999;16:87-9.

10. Zaror L, Aliaga X. Dermatophytes in healthy Chilians. Mycoses. 1990;33:95-8.

11. Hay RJ, Korting HC, Jones TC. Single-dose treatment of athlete's foot with terbinafine: report from a dermatology expert panel. Expert Rev Dermatol. 2007;2:133-8.

12. Ortonne JP, de Chauvin MF, Korting HC, Ghannoum MA, Elewski BE, Hay RJ, Mallefet P, Cahen LP, Grouin JM. Metaanalyses comparing a single application of terbinafine $1 \%$ filmforming solution with other topical treatments for tinea pedis. Eur Dermatol. 2009;4:19-28. 